\title{
Recomendaciones para el manejo de pacientes con enfermedad Inflamatoria Intestinal (EII) y COVID-19
}

\author{
Carlos Agüero L. ${ }^{1,2,7}$, Rodrigo Quera P. ${ }^{3}$, Joselyn Slimming A. ${ }^{4}$, Gonzalo Pizarro J. ${ }^{3}$, \\ Daniela Simian M. ${ }^{3}$, Manuel Álvarez L. ${ }^{1}$, Carolina Pavez O. ${ }^{1}$, Ignacio Alfaro P. ${ }^{5,6}$, \\ María Vergara A. ${ }^{7}$, Regina Hernández $P^{8}$ y Jaime Lubascher $C^{3}$
}

\section{Recommendations for the management of patients with Inflammatory Bowel Disease (IBS) and COVID-19}

The new Coronavirus (SARS-CoV-2) appeared in China in December 2019. Since then and until April 2020 it spread worldwide affecting more than two million people. Its exponential rise is still growing all over the world, taking thousands of lives. SARS-CoV-2 is very contagious, person to person, by droplets which can generate a respiratory infection known as COVID-19. Some patients are at higher risk: Older people, those with cardiovascular disease, diabetes and hypertension are the most prone to an unfavorable outcome. Our Inflammatory Bowel Disease (IBD) patients are a special cluster, with many of them taking immunosuppressive treatment for long periods, which could pose an important risk. Scientifics societies all over the world have joined efforts to generate data, share experiences and make recommendations for good clinical management. This is a review of the available evidence, expert opinion, and proposed ways of working during the pandemic

Key words:

\section{Resumen}

El nuevo coronavirus (SARS-CoV-2) apareció en China en diciembre de 2019. Desde su inicio hasta abril de 2020 se ha expandido por todo el mundo, afectando a más de dos millones de personas. Su ascenso exponencial sigue creciendo, generando miles de muertes. Su contagiosidad es persona a persona por gotitas, pudiendo llegar a generar un cuadro clínico de infección respiratoria conocido como COVID-19. Algunos pacientes tienen más riesgos de tener un curso desfavorable; adultos mayores, pacientes con enfermedad cardiovascular, hipertensos y diabéticos. Nuestros pacientes con enfermedad inflamatoria intestinal son un grupo de pacientes con características particulares, muchos de ellos reciben tratamiento inmunosupresor por largos períodos, lo que pudiese suponer un riesgo específico. Las sociedades científicas de Europa y Norteamérica han realizado un esfuerzo conjunto para generar datos, compartir experiencias y hacer recomendaciones de buen manejo clínico. Esta es una revisión de la evidencia disponible, opiniones de expertos y formas de trabajo propuestos durante la pandemia.

Palabras clave:
Desde diciembre del año 2019, SARS-CoV-2 (Severe Acute Respiratory Syndrome-Coronavirus 2) ha iniciado una escalada de contagio de magnitud global: es un virus contagioso que puede causar enfermedades pulmonares graves y muerte. El contagio es persona a persona, pudiendo infectar al menos a dos personas más. La infección se produce principalmente por gotitas que pueden viajar hasta un metro de distancia y depositarse en superficies.
La infección puede permanecer asintomática o evolucionar con síntomas leves como fiebre, tos, fatiga, dolor muscular e incluso dificultad respiratoria. El test disponible para su diagnóstico es la reacción en cadena de la polimerasa (PCR) disponible en hospitales públicos e instituciones de salud privadas.

La mayoría de los pacientes presentan síntomas leves y se curan de la enfermedad, sin embargo, algunos evolucionan desfavorablemente requiriendo

\author{
Departamento \\ Gastroenterología \\ Facultad de Medicina, \\ Pontificia Universidad \\ Católica de Chile. \\ Santiago, Chile. \\ ${ }^{2}$ Unidad de \\ Endoscopia, Hospital \\ Dr. Gustavo Fricke. \\ Viña del Mar, Chile. \\ ${ }^{3}$ Departamento \\ Gastroenterología \\ Clínica Las Condes. \\ Santiago, Chile. \\ ${ }^{4}$ Clínica Santa María. \\ Santiago, Chile. \\ ${ }^{5}$ Hospital Guillermo \\ Grant Benavente, \\ Concepción, Chile \\ ${ }^{6}$ Clínica Sanatorio \\ Alemán. Concepción, \\ Chile. \\ ${ }^{7}$ Clínica Ciudad del \\ Mar. Viña del Mar, \\ Chile \\ ${ }^{8}$ Clínica Elqui. La \\ Serena, Chile.
}

Recibido:

Aceptado:

Correspondencia a: Dr. Carlos Agüero Luengo

carlosagueroluengo@ gmail.com 
hospitalización para observación (aproximadamente $5 \%$ ) y en una minoría de los casos (4 a 5\%) deben entrar a una unidad de cuidados intensivos para soporte ventilatorio avanzado (ventilación mecánica).

Los pacientes de mayor riesgo son los mayores de 55 años, aquellos con comorbilidades y potencialmente los que toman medicamentos inmunomoduladores y/o tratamiento biológico.

Muchos pacientes con EII, Colitis Ulcerosa o Enfermedad de Crohn, usan tratamientos de mantención con fármacos inmunomoduladores como azatioprina, mercaptopurina o metotrexato; o tratamiento biológico, como adalimumab, infliximab, golimumab o ustekinumab, que les permiten mejorar su calidad de vida, estar en remisión clínica y/o endoscópica.

A continuación entregamos recomendaciones generales a todos los pacientes con EII según contexto clínico y fármacos utilizados. Estas recomendaciones se han basado en el aprendizaje constante del comportamiento del virus en pacientes con EII en otros países y las indicaciones de sociedades científicas internacionales, por lo que podrían ser reevaluadas en futuras versiones según la evidencia disponible.

La Enfermedad Inflamatoria Intestinal (EII), siendo la Colitis ulcerosa (CU) y Enfermedad de Crohn (EC) sus principales exponentes, son enfermedades de curso crónico cuya etiología es aún desconocida aunque influyen en su patogenia factores genéticos, el ambiente, la microbiota y la desregulación del sistema inmune, produciendo una inflamación descontrolada de diversos segmentos del tubo digestivo, las cuales van asociadas en ciertas ocasiones con manifestaciones extraintestinales. El control de la inflamación requiere la supervisión protocolizada de equipos multidisciplinarios y el control del sistema inmune con fármacos inmunosupresores ${ }^{1}$.

En el mundo se ha descrito un aumento sostenido de las tasas de prevalencia e incidencia de EII siendo más significativo en países industrializados ${ }^{2}$. Aunque en Chile no contamos con datos sobre la incidencia y prevalencia de la EII, estudios realizados en centros terciarios confirman un aumento en el número de casos nuevos diagnosticados durante los últimos años ${ }^{3} \mathrm{y}$ en el número de hospitalizaciones por esta patología ${ }^{4}$.

En diciembre de 2019 se inició en China la expansión de un virus conocido posteriormente como SARS-CoV-2, de alta contagiosidad, con la potencialidad de producir un cuadro clínico de insuficiencia respiratoria leve a grave, llegando en algunos casos a requerir apoyo con ventilación mecánica, conocido como COVID-19 (Coronavirus Disease 2019). Producto de la rápida expansión del virus en todo el mundo y las consecuencias humanitarias, la Organización Mundial de la Salud (OMS) declaró el estado de pandemia el 11 de marzo de $2020^{5}$. Al momento de escribir este artículo hay en el mundo 2.284 .018 pa- cientes infectados con 156.901 muertes a nivel global $(6 \%)$ y una curva aún en crecimiento exponencial ${ }^{6}$.

Los pacientes con mayor riesgo de mortalidad son los adultos mayores y aquellos con comorbilidades cardiovasculares, hipertensión arterial y diabetes ${ }^{7}$. El riesgo de los pacientes inmunosuprimidos es aún desconocido, pero se presume que al igual que en otras patologías infecciosas, son un grupo de importante riesgo para infección por SARS-CoV-2 y eventualmente COVID- $19^{8}$.

Los pacientes con EII utilizan medicamentos inmunosupresores (corticoesteroides, terapia biológica como anti-TNF $\alpha$, anti-integrinas y anti p40 IL-12/23 y recientemente moléculas pequeñas) que permiten controlar la actividad inflamatoria y los riesgos asociados de colectomía y muerte, sin embargo, se han descrito una serie de efectos adversos como infecciones virales no COVID-19 y reacciones idiosincráticas que deben ser vigiladas de manera cercana por el equipo tratante ${ }^{9}$. Los riesgos para nuestros pacientes no son solo los derivados de la infección por SARSCoV-2, sino también aquellos generados por la redistribución de los espacios de atención clínica directa, lo que lleva a la postergación de sus controles. Si bien es necesario mantener a los pacientes alejados de los centros de atención de salud, debemos prevenir y evitar el desarrollo de crisis por la falta de información, control o un tratamiento adecuado y oportuno.

Dada la magnitud e impacto mundial de la infección, la ausencia de datos clínicos en pacientes con EII y los posibles riesgos asociados, se están desarrollando esfuerzos colaborativos para generar bases de datos globales y guías de recomendación para el manejo de los pacientes en diversas situaciones clínicas.

El registro mundial de pacientes con EII y COVID-19 que es Surveillance Epidemiology of Coronavirus Under Research Exclusion (SECURE)-IBD permite que cualquier médico ingrese pacientes. Al momento de esta revisión (17.04.2020) habían sido incluidos 580 casos, 1 de Chile, 52\% hombres, 60\% con EC, 58\% en remisión, 109 mayores de 60 años (19\%), 175 (30\%) hospitalizados, 31 (5\%) en UCI y $20(3 \%)$ fallecidos. De los pacientes que fallecieron, 13 eran mayores de 60 años, 10 estaban en remisión ( $\sin$ especificar si ésta consistía solo en remisión clínica o asociada a remisión endoscópica), 12 utilizaban aminosalicilatos, 6 corticoides, 1 azatioprina, 4 anti TNF y 1 inhibidor de JAK kinasa. A pesar de ser datos preliminares, se observa mayor mortalidad en los grupos de riesgo conocidos, adultos mayores y hombres. En relación a fármacos no se puede inferir alguna tendencia particular de riesgo en infección o mortalidad. En la Tabla 1 se muestran las características de pacientes infectados, hospitalizados y fallecidos y en la Tabla 2 la descripción de los fármacos utilizados ${ }^{10}$. 
Tabla 1. Características de pacientes infectados, hospitalizados y fallecidos

\begin{tabular}{|lccc|}
\hline & $\begin{array}{c}\text { Infectados } \\
(\mathbf{n} / \%)\end{array}$ & $\begin{array}{c}\text { Hospitalizados } \\
\mathbf{1 7 5}(\mathbf{n})\end{array}$ & $\begin{array}{c}\text { Fallecidos } \\
\mathbf{2 0}(\mathbf{n})\end{array}$ \\
\hline Hombres & $300(51,7)$ & 102 & 14 \\
E. Crohn & $346(59,6)$ & 94 & 7 \\
\hline Remisión & $337(58,1)$ & 82 & 10 \\
0 - 30 años & $154(26,9)$ & 27 & 0 \\
\hline $31-60$ años & $314(54,4)$ & 89 & 7 \\
\hline$>60$ años & $109(18,7)$ & 59 & 13 \\
\hline
\end{tabular}

Datos al 04.17.20. Adaptado de SECURE-IBD Database Public Data Update. covidibd.org

El objetivo de este documento es entregar una guía de las recomendaciones realizadas por diferentes sociedades médicas y consensos de expertos con el fin optimizar el control y tratamiento de nuestros pacientes con EII durante la pandemia. Tanto la Organización Europea de Crohn y Colitis (ECCO) y la Organización Internacional para el Estudio de las Enfermedades Inflamatorias Intestinales (IOIBD) han generado grupos de trabajo y han realizado recomendaciones que presentamos a continuación.

1. Educar a los pacientes en el autocuidado en diversas circunstancias. Evidencia reciente de educación temprana a los pacientes con EII ha demostrado ser efectiva en China. Trescientos dieciocho pacientes con EII que fueron instruidos en su cuidado y seguidos en la evolución de la epidemia en Hubei mostró que ninguno tuvo infección o enfermedad reportada en el seguimiento ${ }^{11}$. ACCTECU (Agrupación Chilena de Trabajo Enfermedad de Crohn y Colitis Ulcerosa) ha desarrollado una guía de recomendaciones para pacientes de libre disposición que se adjuntan en Anexo $\mathrm{N}^{\circ} 1$ y que está disponible para descarga en la página de la Sociedad Chilena de Gastroenterología (www. sociedadgastro.cl).

2. La guía de la Sociedad Británica de Gastroenterología propone clasificar a los pacientes de acuerdo a su riesgo ya sea en alto, moderado o leve según su edad, comorbilidad y características de la EII ${ }^{12}$ y promover el aislamiento "tipo escudo" (sin ningún tipo de contacto social a los de riesgo alto, aislamiento social estricto a los de riesgo moderado y aislamiento físico a los de riesgo leve (Tabla 3).

3. Es importante definir estrategias que permitan contactar a los pacientes con EII, privilegiando a aquellos de mayor riesgo, educarlos en relación a sus cuidados y prevención. Se debe evitar la
Tabla 2. Características farmacológicas de infectados, hospitalizados y fallecidos

\begin{tabular}{|lccc|}
\hline Fármacos & $\begin{array}{c}\text { Infectados } \\
\mathbf{5 8 0}(\mathbf{n}, \mathbf{\%})\end{array}$ & $\begin{array}{r}\text { Hospitalizados } \\
(\mathbf{n})\end{array}$ & $\begin{array}{c}\text { Fallecidos } \\
\mathbf{2 0}(\mathbf{n})\end{array}$ \\
\hline 5 ASA & $137(23,6)$ & 64 & 12 \\
Corticoides & $67(11,5)$ & 41 & 6 \\
AZA/6 MP & $58(10)$ & 25 & 1 \\
MTX & $5(0,86)$ & 3 & 0 \\
Anti TNF sin AZA/6MP/MTX & $191(32,9)$ & 28 & 2 \\
Anti TNF + AZA/6MP/MTX & $56(9,6)$ & 24 & 2 \\
Antiintegrinas & $54(9,3)$ & 16 & 0 \\
Inhibidor de JAK & $8(1,37)$ & 1 & 1 \\
Inhibidor IL 12/23 & $64(11)$ & 5 & 0 \\
\hline
\end{tabular}

AZA (azatioprina), 6MP (6-mercaptopurina), MTX (metotrexato), JAK (jak kinasas). Datos al 04.17.20. Adaptado de SECURE-IBD Database Public Data Update. covidibd.org

consulta directa y facilitar los espacios para tener contacto telefónico o por telemedicina. En casos de crisis, los pacientes deben ser evaluados de forma precoz y así evitar mayor riesgo de infección, uso de corticoides, hospitalización y cirugía.

4. El control clínico de pacientes asintomáticos y/o con crisis leve se debe realizar por métodos no presenciales (telemedicina, teléfono) y la evaluación de la severidad con índices clínicos de actividad. Se podría considerar la medición de calprotectina fecal en kits de autoexamen por métodos cromatográficos, evitando al máximo la colonoscopia por riesgos asociados de contagio.

5. Los pacientes con EII y tratamientos inmunomoduladores tienen un riesgo aumentado de influenza y enfermedad neumocócica ${ }^{13}$. La vacunación disminuye la morbilidad y mortalidad en pacientes con enfermedades autoinmunes en tratamiento inmunosupresor aun cuando sean menos efectivas por el efecto de los fármacos ${ }^{14}$. Con el fin de prevenir otras infecciones pulmonares, en el contexto de una infección COVID-19, todos los pacientes con EII deben ser vacunados contra influenza y neumococo de acuerdo a su esquema habitual.

6. Se debe explicitar a los pacientes que no deben automedicarse con corticoides dado los riesgos de infecciones oportunistas como $P$. jiroveci y una evolución desfavorable en caso de infección pulmonar COVID-19.

7. En la pandemia COVID-19 la diarrea se ha presentado de forma variable y precoz entre el 2 y $33 \%$ de los pacientes, pudiendo aislarse material genético de las heces sin claro rol patológico ni 
Tabla 3. Clasificación de riesgo pacientes con EII

\begin{tabular}{|c|c|c|}
\hline Riesgo alto & $\begin{array}{l}\text { Riesgo Moderado } \\
\text { Tratamiento inmunomodulador }\end{array}$ & Riesgo leve \\
\hline $\begin{array}{l}\text { - Mayores } 70 \text { años } \\
\text { - terapia inmunomoduladora } \\
\text { - Enf. Cardiopulmonar } \\
\text { - Diabetes, HTA } \\
\text { Inicio reciente de anti TNF + inmunomodulador } \\
\text { o corticoides } \\
\text { - Cualquier edad } \\
\text { + Corticoides (> } 20 \text { mg prednisona por un } \\
\text { período de más de } 2 \text { semanas) } \\
\text { + Inicio anti TNF con inmunosupresor (6 sem) } \\
\text { + Crisis moderada, severa sin respuesta a } \\
\text { tratamiento } \\
\text { + Intestino corto con NPT } \\
\text { + Necesidad de NPT }\end{array}$ & $\begin{array}{l}\text { - Anti TNF } \\
\text { - Biológico + inmunomodulador en } \\
\text { paciente estable } \\
\text { - Ustekinumab } \\
\text { - Vedolizumab } \\
\text { - Tiopurinas } \\
\text { - Metotrexato } \\
\text { - Inh. Calcineurina } \\
\text { - Inh. Janus Kinasa } \\
\text { - Micofenolato, tacrolimus } \\
\text { - Talidomida } \\
\text { - Prednisona }<20 \mathrm{mg} / \text { día }\end{array}$ & $\begin{array}{l}\text { - Aminosalicilatos } \\
\text { - Terapias rectales } \\
\text { - Corticoides orales tópicos (budesonida) } \\
\text { - Colestiramina } \\
\text { - Antidiarreicos } \\
\text { - Antibióticos } \\
\text { - Probióticos }\end{array}$ \\
\hline
\end{tabular}

Adaptada de Kennedy NA. Gut, abril $2020^{12}$.

riesgo de transmisión ${ }^{15,16}$. En el caso del virus influenza H1N1v, se ha demostrado el desarrollo de crisis leves en pacientes con Colitis Ulcerosa. ${ }^{17}$ Aunque hasta la fecha no hay clara evidencia que SARS-CoV-2 puede gatillar una crisis, debemos estar alertas a la evolución con fiebre y síntomas respiratorios.

8. En pacientes sin diagnóstico previo de EII y sintomáticos moderados a severos, se puede realizar una colonoscopia manteniendo las normas de EPP según las guías de la Asociación Chilena de Endoscopia Digestiva (ACHED) y del centro endoscópico local.

9. En caso de una crisis de EII moderada a severa, el paciente se debe poner en contacto con el médico o equipo local para informar características del cuadro actual y definir en conjunto el mejor lugar de atención. Es importante que el equipo tratante conozca los servicios de atención de urgencia y las características de atención locales para orientar a los pacientes de forma adecuada.

10. Todos los procedimientos complementarios al estudio y control de los pacientes con EII; ya sea estudios endoscópicos, imagenológicos, de laboratorio y quirúrgicos no urgentes deben ser valorados con juicio clínico de acuerdo a su beneficio inmediato, riesgo vital y situación local de la pandemia.

11. Los tratamientos farmacológicos deben ser indicados considerando el riesgo de inmunosupresión e infección con la posibilidad de tener una crisis de la enfermedad. Es muy importante NO SUSPENDER EL TRATAMIENTO INMUNOSUPRESOR O BIOLÓGICO en curso a menos que exista una contraindicación formal o un cuadro infeccioso intercurrente. Monitorizar las dosis y sus efectos adversos a través de medios no presenciales, siempre que sea posible y conocer algunas consideraciones específicas definidas a continuación. Es importante considerar las vidas medias de los fármacos ya que son necesarias 5 6 vidas medias para lograr tener niveles bajos de los fármacos.

\section{Aminosalicilatos (5 ASA)}

- No hay evidencias de que su uso aumente el riesgo de COVID-19 o la severidad de ésta.

- En pacientes sintomáticos con dosis insuficientes, se debe optimizar el tratamiento siempre que sea posible, controlando su respuesta clínica e idealmente con calprotectina fecal con el fin de asegurar la curación de mucosa y prevenir crisis.

\section{Corticoides}

- Evitarlos siempre que sea posible. En caso de ser necesario, es importante mantener a los pacientes en aislamiento social estricto mientras las dosis de prednisona sean superiores a $20 \mathrm{mg}$ o su equivalente. 
- Las dosis altas de corticoides son de alto riesgo para infecciones respiratorias, oportunistas y sepsis $^{18,19}$.

- Disminución de los corticoides debe ser lo más rápido posible. Considerar disminuir $10 \mathrm{mg} / \mathrm{kg}$ por semana balanceando el riesgo de infección con posibilidad de reactivación. Considerar control clínico proactivo para definir terapia de mantención.

- Nunca deben ser suspendidos abruptamente.

- Considerar el uso de corticoides de uso tópico (enemas o supositorios).

- Budesonida (9 mg/día por 8 a 12 semanas.) en pacientes con EC y compromiso de intestino delgado y/o colon ascendente. En pacientes con $\mathrm{CU}$ se puede usar o budesonida MMX $(9 \mathrm{mg} /$ día por 8 a 12 semanas). Considerar control clínico a las 2 semanas para definir terapia de mantención.

- Cuando COVID-19 evoluciona severamente se puede producir un síndrome de liberación de citoquinas. Interleuquina-6 (IL6) e interleuquina2R (IL2R) están altamente expresadas en estos pacientes por lo que se ha propuesto el tratamiento con Tozilizumab (NCT04306705) con buenos resultados iniciales. El uso de pequeños pulsos de corticoides en este escenario $(0,5-1 \mathrm{mg} / \mathrm{kg}$ por 7 días) pudiese ser beneficioso y se deben evaluar los riesgos versus los beneficios ${ }^{20}$.

\section{Inmunomoduladores: tiopurínicos (azatioprina, 6-mercaptopurina), metotrexato, tacrolimus}

- Tiopurínicos: No existen claras evidencias que aumenten el riesgo de tener COVID-19. Sin embargo, la disminución de linfocitos T CD4 podría condicionar una eliminación más lenta del virus. En caso de COVID-19 parece razonable suspender y reiniciar 21 a 30 días después de la mejoría clínica. Es posible que en futuros escenarios sea necesario examen de PCR y/o anticuerpos para reiniciar tratamiento. Evaluar riesgo beneficio como terapia inicial en tiempos de pandemia, particularmente en pacientes de alto riesgo. Considerar que su efecto no aparece antes de las 8 a 12 semanas y como su vida media es de 6,8 días, logra niveles muy bajos a los 40 días desde la suspensión.

- Metotrexato. El mecanismo de acción no debería condicionar la evolución ni la mejoría clínica, sin embargo parece razonable suspender su administración durante el cuadro clínico de infección por COVID-19 y reiniciar 21 a 30 días después de la mejoría clínica. Considerar que su vida media es de $6 \mathrm{~h}$ tomando menos de 3 días para eliminar el fármaco.
Anti TNF: Infliximab, adalimumab, golimumab, certolizumab

- No hay evidencias que su uso aumente el riesgo de tener COVID-19.

- Considerar uso inicial en monoterapia subcutánea para evitar riesgo de inmunogenicidad en inicio de tratamiento. Además, el inicio de infliximab implicará la necesidad de asistir a un centro asistencial público o privado para su administración.

- Control con niveles del fármaco según respuesta clínica y disponibilidad para ajustar terapia en la medida de lo posible.

- No cambiar terapia endovenosa por terapia subcutánea. Se ha descrito riesgo de pérdida de respuesta en cambios electivos de pacientes con $\mathrm{EC}^{21}$.

- En caso de tener un paciente COVID-19 se sugiere suspender tratamiento y posponer la próxima dosis para 21-30 días después de la mejoría clínica.

- En caso de suspender considerar vidas medias: Adalimumab 10-20 días; Infliximab 7-12 días.

\section{Inhibidores de Interleukina 12/23:} Ustequinumab

- No hay evidencias que su uso aumente el riesgo de enfermedad por COVID-19.

- Su principal ventaja es la facilidad de uso subcutáneo después de la primera dosis endovenosa.

- En caso de tener un paciente COVID-19 se sugiere suspender tratamiento y posponer la próxima dosis para 21-30 días después de la mejoría clínica.

- En caso de suspender considerar vida media de 19 días.

\section{Anti Integrina Alfa 4 Beta 7: Vedolizumab}

- Sin evidencias que su uso aumente el riesgo de infección.

- En caso de tener un paciente COVID-19 se sugiere suspender tratamiento y posponer la próxima dosis para 21-30 días después de la mejoría clínica.

\section{Inhibidores de Janus Kinasa: Tofacitinib}

- Sin evidencias que su uso aumente el riego de infección.

- En caso de tener un paciente COVID-19 se sugiere suspender tratamiento y posponer la próxima dosis para 21-30 días después de la mejoría clínica.

Nota: Las recomendaciones de esta guía han sido realizadas de acuerdo a la evidencia clínica disponible y serán reevaluadas según nuevas publicaciones y experiencias clínicas locales. 


\section{Artículo Original}

Anexo 1. Recomendaciones para pacientes: Enfermedad Inflamatoria Intestinal (EII) Pandemia por COVID-19 2020 - Versión 2/17 de abril de 2020

Coronavirus (SARS-CoV-2) es un virus contagioso que puede causar enfermedades pulmonares severas y muerte. (COVID-19). El contagio es persona a persona, pudiendo infectar al menos a dos personas más. La infección se produce principalmente por gotitas que pueden viajar hasta un metro de distancia y depositarse en superficies.

La infección puede permanecer asintomática o evolucionar con síntomas leves como fiebre, tos, fatiga, dolor muscular e incluso dificultad respiratoria. El test disponible para su diagnóstico es la reacción en cadena de la polimerasa (PCR) disponible en hospitales públicos e instituciones de salud privadas.

La mayoría de los pacientes presentan síntomas leves y se curan de la enfermedad, sin embargo, algunos evolucionan desfavorablemente, requiriendo hospitalización para observación (aproximadamente 5\%) y en una minoría de los casos (4 a 5\%) deben entrar a una unidad de cuidados intensivos para soporte ventilatorio avanzado (ventilación mecánica).

Los pacientes de mayor riesgo son los mayores de 55 años, aquellos con comorbilidades y potencialmente los que toman medicamentos inmunomoduladores y/o tratamiento biológico.

Muchos pacientes con EII, Colitis Ulcerosa o Enfermedad de Crohn, usan tratamientos de mantención con fármacos inmunomoduladores como azatioprina, mercaptopurina o metotrexato; o tratamiento biológico, como adalimumab, infliximab, golimumab o ustekinumab, que les permiten mejorar su calidad de vida, estar en remisión clínica y/o endoscópica.

A continuación, entregamos recomendaciones generales a todos los pacientes con EII según contexto clínico y fármacos utilizados. Estas recomendaciones se han basado en el aprendizaje constante del comportamiento del virus en pacientes con EII en otros países y las indicaciones de sociedades científicas internacionales por lo que podrían ser reevaluadas en futuras versiones según la evidencia disponible.

Estas recomendaciones no reemplazan el control con médico de referencia.

\section{Recomendación diaria universal para todos los pacientes con EII}

- No suspender tratamiento.

- Vacuna estacional de influenza.

- Vacuna neumocócica (Prevenar 13 y/o Pneumo 23).

- No viajar, dentro o fuera del país.

- Mantener aislamiento en casa el máximo posible, evitar lugares con acumulación de personas. Contacto social mayor a 1 metro de distancia.

- No saludar de mano, besos o abrazos.

- Evitar contacto con personas que están enfermas; con o sin COVID-19.

- Evitar tocarse la cara, incluyendo nariz, boca y ojos.

- Lavar manos con jabón varias veces al día por 20 segundos y siempre después de ir al baño, antes de comer y después de sonarse la nariz, toser o estornudar.

- Evitar contacto directo con todo tipo de superficies en espacios públicos (como barandas, manillas) Si lo realiza, debe lavar sus manos con agua y jabón por al menos 20 segundos.

- Si no puede lavar manos con jabón, usar algún sanitizador con $60 \%$ de alcohol.

- En casa lavar y limpiar recurrentemente las superficies, idealmente con cloro.

- Lavar diariamente WC con cloro ya que existe eliminación del virus en las deposiciones.

- Tenga un plan en caso de enfermar.

Recomendaciones extras para pacientes de riesgo moderado y alto

Mayores de 60 años, enfermedades cardiopulmonares, HTA y diabetes

Tratamiento inmunomodulador y/o biológico

- Distanciamiento social estricto. Permanecer en casa, restringiendo el ingreso de personas (Nadie sale, nadie entra).

- No suspender el tratamiento. Los medicamentos no aumentan el riesgo de contraer COVID-19.

- Estar en contacto con equipo médico tratante.

- Uso de corticoides es exclusivamente de indicación médica, no los inicie por su cuenta, ya que aumenta el riesgo de complicaciones al enfermar con COVID-19. Si Ud. ya ha iniciado tratamiento con corticoides por indicación médica, no modifique la dosis ni los suspenda; pónganse en contacto con su médico antes de realizar modificaciones.

Recomendaciones para pacientes sintomáticos

- Si Ud. presenta fiebre, tos o dificultad respiratoria, debe acudir a un servicio de urgencia, para descartar infección con COVID-19.

- No usar antiinflamatorios orales.

- Si se confirma su diagnóstico, siga las instrucciones del equipo médico que lo esté atendiendo. Póngase en contacto con su médico tratante, quien lleva su tratamiento por EII. Hágale saber su diagnóstico para ajustar los tratamientos de acuerdo a su condición clínica.

- Todos sus contactos estrechos deberán iniciar cuarentena preventiva.

En caso de dudas llamar a salud responde al fono 6003607777 o visitar el sitio www.saludresponde.cl donde podrá solicitar la llamada.

Para más información ingresar a: https://www.minsal.cl/nuevo-coronavirus-2019-ncov/

https://www.who.int/es/emergencies/diseases/novel-coronavirus-2019 


\section{Referencias}

1.- Schirbel A, Fiocchi C. Inflammatory bowel disease: Established and evolving considerations on its etiopathogenesis and therapy. J Dig Dis 2010;11:266-76.

2.- Molodecky NA, Soon IS, Rabi DM, Ghali WA, Ferris M, Chernoff G. Increasing incidence and prevalence of the inflammatory bowel diseases with time, based on systematic review. Gastroenterology 2012;142:46-54.

3.- Simian D, Fluxá D, Flores L. Lubascher J, Ibáñez P, Figueroa C, et al. Inflammatory Bowel Disease: A descriptive study of 716 local Chilean patients. World J Gastroenterol 2016;22:5267-75.

4.- Bellolio Roth F, Gómez J, Cerda J. Increase in Hospital Discharges for Inflammatory Bowel Diseases in Chile Between 2001 and 2012. Dig Dis Sci 2017;62:2311-7.

5.- Ghebreyesus T. WHO Director-General's opening remarks at the media briefing on COVID-19 - 11 March 2020. https:// www.who.int/dg/speeches/detail/whodirector- general-s-opening-remarks-atthe-media-briefing-on-covid-19---11march-2020 (Consultado el 29 de marzo de 2020).

6.- Johns Hopkins Center for Systems Science and Engineering. Coronavirus COVID-19 Global Cases by Johns Hopkins CSSE. https://coronavirus.jhu. edu/ (Consultado el 18 de abril de 2020).

7.- Onder G, Rezza G, Brusaferro S. Case-Fatality Rate and Characteristics of Patients Dying in Relation to COVID-19 in Italy. JAMA. 2020 Mar 23. doi: 10.1001/jama.2020.4683. [Epub ahead of print].
8.- Rubin DT, Abreu MT, Rai V, Siegel CA, International Organization for the Study of Inflammatory Bowel Disease, Management of Patients with Crohn's Disease and Ulcerative Colitis During the COVID-19 Pandemic: Results of an International Meeting. Gastroenterology 2020. Apr 6. pii: S0016-5085(20)30465.

9.- Wisniewski A, Kirchgesner J, Seksik P, Landman C, Bourrier A, Nion-Larmurier $\mathrm{I}$, et al. Increased incidence of serious viral infections with inflammatory bowel disease associates with active disease and use of thiopurines. United European Gastroenterology Journal 2020. https:// doi.org/10.1177/2050640619889763.

10.- Brenner EJ, Ungaro RC, Colombel JF, Kappelman MD. SECURE-IBD Database Public Data Update. covidibd.org. Consultado el 17 de abril de 2020.

11.- An P, Ji M, Ren H, Su J, Kang J, Yin A, et al. Protection of 318 inflammatory bowel disease patients from the outbreak and rapid spread of COVID-19 infection in Wuhan, China. Disponible en SSRN: https://ssrn.com/abstract $=3543590$ or http://dx.doi.org/10.2139/ssrn.3543590.

12.- Kennedy NA, Jones GR, Lamb CA, Appleby R, Arnott I, Beattie RM, et al. British Society of Gastroenterology guidance for management of inflammatory bowel disease during the COVID-19 pandemic. Gut Epub ahead of print: [18.04.20]. doi: 10.1136/ gutjnl-2020-321244.

13.- Tinsley A, Navabi S, Williams ED, Liu G, Kong L, Coates MD, et al. Increased Risk of Influenza and Influenza-Related Complications Among 140,480 Patients With Inflammatory Bowel Disease. Inflamm Bowel Dis 2019;25:369-76.

14.- Rahier JF, Magro F, Abreu C, Armuzzi A,
Ben-Horin S, Chowers Y, et al. Second European evidence-based consensus on the prevention, diagnosis and management of opportunistic infections in inflammatory bowel disease. J Crohns Colitis. 2014 8:443-68.

15.- Liang W, Feng Z, Rao S, Xiao C, Xue $X$, Lin Z, et al. Diarrhoea may be underestimated: a missing link in 2019 novel coronavirus. Gut 2020 Feb 26 doi:10.110 1/2020.02.03.20020289.

16.- Holshue ML, DeBolt C, Lindquist S, Lofy $\mathrm{KH}$, Wiesman J, Bruce H, et al. First case of 2019 novel coronavirus in the United States. N Engl J Med 2020; 382:929-36.

17.- Rahier JF, Papay P, Salleron J, Sebastian $\mathrm{S}$, Ellul P, Teich N, et al. Influenza $\mathrm{A}(\mathrm{H} 1 \mathrm{~N} 1) \mathrm{v}$ infection in patients with inflammatory bowel disease: a case series. Aliment Pharmacol Ther 2011;33:499-500.

18.- Naganuma M1, Kunisaki R, Yoshimura N, Takeuchi Y, Watanabe M. A prospective analysis of the incidence of and risk factors for opportunistic infections in patients with inflammatory bowel disease. J Gastroenterol. 2013;48:595-600.

19.- Fardet L, Petersen I, Nazareth I. Common infections in patients prescribed systemic glucocorticoids in primary care: a population-based cohort study. PLoS Med 2016; 13.

20.- Shang L, Zhao J, Hu Y, Du R, Cao B. On the use of corticosteroids for $2019-\mathrm{nCoV}$ pneumonia. Lancet. 2020;395:683-4.

21.- Hoentjen F, Haarhuis BJ, Drenth JP, de Jong DJ. Elective switching from infliximab to adalimumab in stable Crohn's disease. Inflamm Bowel Dis. 2013;19:761-6. 